\title{
The Leading Blastomere of the 2-Cell Stage Parthenogenetic Porcine Embryo Contributes to the Abembryonic Part First
}

\author{
Sang Kyu PARK ${ }^{1) *}$, Cheolhee $\mathrm{WON}^{1) *}$, Young-Ju CHOI ${ }^{1)}$, Hoin $\mathrm{KANG}^{1)}$ and Sangho $\mathrm{ROH}^{1) * *}$ \\ ${ }^{1)}$ Embryo Biotechnology Laboratory, CLS21 and Dental Research Institute, Seoul National University School of Dentistry, Republic of \\ Korea
}

(Received 1 April 2008/Accepted 1 December 2008) ABSTRACT. Polarity formation in preimplantation embryos is controversial. To investigate the embryonic-abembryonic axis in the pig, por-
cine parthenotes were used to prevent the topological change caused by polyspermy as well as to avoid the influences of sperm entry
position. For lineage tracing, DiI, a fluorescence dye, was injected into only a blastomere of the 2-cell stage embryos. If the first blas-
tomere to divide was labeled, the embryo was included in the leading group, and while all others were included in the lagging group.
In $60.5 \%$ of the blastocysts in the lagging group, the progeny of the labeled blastomeres formed the inner cell mass (ICM) and adjacent
trophectoderm (TE) hemisphere; $62.1 \%$ of the blastocysts in the leading group had progeny of the labeled blastomeres distributed only
to the TE (opposite of ICM). The rest of the lagging and leading groups showed random distributions. Unlike murine parthenotes, biased
mitochondrial distribution was also found in porcine parthenotes ( $38.1 \%$ ). Our findings indicate that the 'leading' blastomere of the 2 -
cell porcine parthenote forms the distal TE (abembryonic) and that the 'lagging' blastomere forms the remaining portion of the blastocyst,
including the ICM (embryonic). Biased distribution of mitochondria in each 2-cell blastomere may contribute partly to this event.
KEY wORDS: embryonic-abembryonic polarity, mitochondria, parthenogenesis, porcine.

Proper embryo polarity is a prerequisite factor for normal animal development. Most vertebrate and invertebrate embryos display undeniable polarity along a plane known as the animal-vegetal axis. In Drosophila, embryonic axes are specified during oogenesis, while the true embryonic axes of the vertebrate embryo are established after fertilization [12]. In species such as frogs, information relating to specification of the future body axis is asymmetrically localized as stored maternal factors in fertilized oocytes [10]. It is also known that the dorsoventral axis of the zebrafish embryo is apparent at the four-cell stage and that the molecular mechanism of axis formation may be evolutionarily conserved [8]. However, early mammalian embryos have long been considered to lack the polarity evident in insects, amphibians and fish before the end of preimplantation because of the ease with which individual blastomeres can be manipulated and used to regenerate entire embryos and to form chimeras [24, 27, 28, 30]. This has led to the long-held assumption that the polarity of most mammalian embryos remains undetermined until the blastocyst stage. The blastocyst contains two types of cells, the clump of cells called the inner cell mass (ICM) and the surrounding cell layer called the trophectoderm (TE). Formation of these two lineages establishes the embryonic-abembryonic (Em-Ab) polarity in the blastocyst. The Em hemisphere contains the polar TE and deeper ICM, or presumptive epiblast. The Ab hemisphere contains the mural TE and surface ICM, or presumptive primitive endoderm. The Em-Ab polarity of the

\footnotetext{
* These authors contributed equally to this work.

**Correspondence to: Roh, S., College of Dentistry, Seoul National University, Jongno-gu, Seoul 110-749, Republic of Korea.

e-mail: sangho@snu.ac.kr
}

embryo corresponds to the dorsal-ventral axis in the postimplantation stage embryo [2]. However, recent studies have proposed that the Em-Ab axis of the mouse blastocyst arises perpendicular to the first cleavage plane [5, 7, 20, 23]. It has been shown that the animal-vegetal axes of mammalian embryos can be traced by the location of the polar body at the animal pole until the blastocyst stage [6]. It has also been claimed that the sperm entry position (SEP) determines the embryonic axis [21], although there has also been a report claiming that the plane of first cleavage is not related to the distribution of the sperm component [4]. In their study, Piotrowska and Zernicka-Goetz (2001) defined the first cleavage plane as passing through both the second polar body and the SEP. Moreover, the same group also claimed that the blastomere in the two-cell stage embryo that inherits the SEP divides earlier than the other blastomere and tends to contribute preferentially to the ICM [21]. Thus, the first cleavage of the mouse zygote predicts the blastocyst axis by breaking the symmetry of the embryo, thereby generating blastomeres with different developmental characteristics [23]. To determine whether sperm entry contributes to the fates of the first two blastomeres or if their fates are an intrinsic property of the oocyte itself, the distribution of the progeny of early blastomeres in parthenogenetic embryos was examined [22]. Unlike fertilized oocytes, no tendency for the first two parthenogenetic blastomeres to follow different fates was found, supporting the conclusion that the fertilization process contributes to setup of embryonic patterning. In contrast with the above claims, recent time-lapse recordings have shown that the first cleavage plane is not predetermined but is defined only by the topology of the two apposing pronuclei in the mouse embryo [9] and that the polarity of the mouse embryo is established at the blastocyst 
stage and is not prepatterned [18, 19]. Controversy still exists as to whether the individual blastomeres from twocell-stage embryos have identical developmental fates. However, most reports have been limited to murine species $[2,7,9,15,23,29]$. The distribution of active mitochondria may be indicative of the energy or ion requirements of many key events during oocyte maturation, fertilization and early embryonic development [26]. If the progeny of the first dividing blastomere tends to contribute preferentially to the ICM as reported previously by another group [21], the first dividing cell may require more active mitochondria in its cytoplasm to obtain more energy for faster development; alternatively biased distribution of mitochondria in each blastomere could affect the order of the next cleavage of 2cell embryos or vice versa.

Here in, we investigated the Em-Ab axis of the blastocyst in the porcine species. Unlike mice, the in vitro maturation (IVM) and fertilization (IVF) processes are the most common and economic choices for obtaining 2-cell embryos in a pig model. However, polyspermic fertilization is a major problem in porcine IVF [14]. To avoid the influences of the fertilization cone, which indicates the sperm entry position, and to prevent topological change of the two or more apposing pronuclei in the egg center caused by polyspermy after IVF, we chose porcine parthenogenetic embryos for use in the present study even though they may not represent normal embryonic development in the pig. This approach may also answer whether the fate of early porcine embryos exists as an intrinsic property of the oocyte itself. Here in, we describe the fate of an individual blastomere from a 2-cellstage parthenogenetic porcine embryo.

\section{MATERIALS AND METHODS}

Porcine oocyte recovery and in vitro maturation: Slaughterhouse ovaries were collected from 5- to 6-month-old prepubertal gilts (body weight: $100 \pm 10 \mathrm{~kg}$ ), placed in saline at $30-35^{\circ} \mathrm{C}$ and transported within $2 \mathrm{hr}$ to our laboratory. After washing with saline three times, cumulus-oocyte complexes (COCs) were recovered by aspiration of 2- to 5-mm follicles using an 18-gauge hypodermic needle attached to a $5-\mathrm{ml}$ disposable syringe. After washing three times in IVM medium, COCs that were enclosed by more than three layers of compact cumulus cells and that had an evenly granulated ooplasm were selected for IVM. The selected COCs were cultured in 4-well culture dishes (Nunc, Denmark) containing $500 \mu \mathrm{l}$ of IVM medium under warmed and gas-equilibrated mineral oil for $44-46 \mathrm{hr}$ at $38.5^{\circ} \mathrm{C}$ in $5 \% \mathrm{CO}_{2}$. The IVM medium for the oocytes was composed of tissue culture medium 199 with Earle's salts and L-glutamine (TCM199, Gibco Life Technologies Inc., U.S.A.) supplemented with $26.2 \mathrm{mM} \mathrm{NaHCO}$, $3.05 \mathrm{mM}$ glucose, $0.91 \mathrm{mM}$ sodium pyruvate, $0.57 \mathrm{mM}$ L-cysteine, $10 \mathrm{ng} / \mathrm{ml}$ epidermal growth factor, $10 \mathrm{IU} / \mathrm{ml}$ equine chorionic gonadotropin and human chorionic gonadotropin and $0.1 \%$ (v/v) PVA [13].

Porcine parthenogenesis and in vitro culture: Electrical activation was performed at room temperature using a CF-
150/B electro-cell fusion system (BLS, Hungary) in a chamber that contained two stainless steel electrodes that were $1.0 \mathrm{~mm}$ apart. The chamber was filled with $0.26 \mathrm{M}$ mannitol supplemented with $0.1 \mathrm{mM} \mathrm{MgSO}_{4}, 0.05 \mathrm{M} \mathrm{CaCl}_{2}$, and $0.01 \%$ PVA. Oocytes were activated with a $1.6 \mathrm{kV} / \mathrm{cm} \mathrm{DC}$ pulse for $40 \mu \mathrm{sec}$ in an activation buffer. To prevent pseudo-polar body extrusion, the activated oocytes were treated for 5 to $6 \mathrm{hr}$ in North Carolina State University-23 medium (NCSU-23) supplemented with $5 \mu \mathrm{g} / \mathrm{ml}$ cytochala$\sin$ B. The oocytes were then washed nine times with cytochalasin B-free NCSU-23 and cultured in 20- $\mu$ l drops (10-15 oocytes per drop) of the same medium for $168 \mathrm{hr}$ at $38.5^{\circ} \mathrm{C}$ in $5 \% \mathrm{CO}_{2}$.

Murine oocyte recovery and parthenogenetic activation: Eight-week-old C57BL6 X DBA2 F1-hybrid mice were superovulated by intraperitoneal injections of 7.5 IU eCG and 7.5 IU hCG given $48 \mathrm{hr}$ apart. Oocytes were recovered $16 \mathrm{hr}$ after hCG injection, and the oviducts were removed and transferred to a Petri dish containing $2 \mathrm{ml}$ Hepes-buffered CZB medium (HCZB) supplemented with $300 \mathrm{IU} / \mathrm{ml}$ hyaluronidase. The oviduct ampullae were opened, and the cumulus-enclosed oocytes were released. After 2 to $3 \mathrm{~min}$ of exposure to the medium, the cumulus-free oocytes were washed twice in HCZB before activation. The recovered oocytes were immediately exposed to an activation medium consisting of $10 \mathrm{mM} \mathrm{SrCl}{ }_{2}$ with $5 \mu \mathrm{g} / \mathrm{ml}$ cytochalasin B in calcium-free CZB for $5 \mathrm{hr}$ and then cultured for $115 \mathrm{hr}$ in $\mathrm{KSOM}$ at $37^{\circ} \mathrm{C}$ in a humidified atmosphere of $5 \% \mathrm{CO}_{2}$ in air.

Fluorescent labeling of each blastomere: In the first stage of the experiment, one blastomere of each 2-cell stage parthenogenetic porcine embryo was randomly labeled with a lipophilic fluorescent red color dye, DiI (1,1'-dioctadecyl3,3,3',3'-tetramethylindocarbocyanine perchlorate; Molecular Probes, Eugene, OR, U.S.A.), by direct injection of the dye into the cytoplasm using an 8- $\mu \mathrm{m}$ inner diameter beveled pipette (Fig. 1A). In some embryos, DiD (blue color dye; 1,1'-dioctadecyl-3,3,3',3'-tetramethylindodicarbocyanine perchlorate) was injected into a blastomere that was not labeled with DiI to examine whether dye injection itself affects the distribution of the progeny of early blastomeres (Fig. 1B). Both dyes were dissolved in virgin olive oil at 2.5 $\mathrm{mg} / \mathrm{ml}$ before use [1]. The labeled embryos were subsequently cultured in NCSU-23 at $38.5^{\circ} \mathrm{C}$ in $5 \% \mathrm{CO}_{2}$. They were monitored every $60 \mathrm{~min}$ until observation of blastomere cleavage. As the oil drop stayed in the cytoplasm until at least the next cleavage, blastomeres containing an oil drop were considered to be labeled blastomeres (Fig. 2). If the blastomere that divided first was labeled, the embryo was included in the 'leading' group; all other embryos were included in the 'lagging' group (Fig. 3). The embryos were then cultured for 6 days in vitro. For nuclear staining of descendant embryos, the blastocysts were washed in $0.1 \%$ PVA-PBS and then permeabilized in PBS supplemented with $10 \mu \mathrm{g} / \mathrm{ml}$ Hoechst\#33342 at room temperature for 20 min. After rinsing in PBS, the embryos were visualized using a confocal laser scanning microscope system (LSM 5 Pascal, Carl Zeiss, Germany or FV300, Olympus, Japan), 

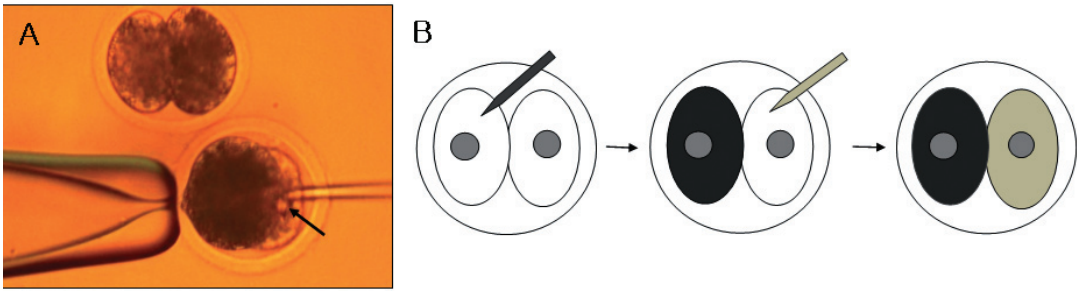

Fig. 1. Fluorescent dye labeling of a blastomere of 2-cell stage porcine parthenote. (A) Oilbased red fluorescent dye, DiI, is released into the cytoplasm of a blastomere. The arrow indicates the released oil drop containing DiI. (B) Illustration of bilateral labeling of 2-cell blastomeres with DiI (red) and DiD (blue). The progeny of each blastomere continues to show either red or blue color.
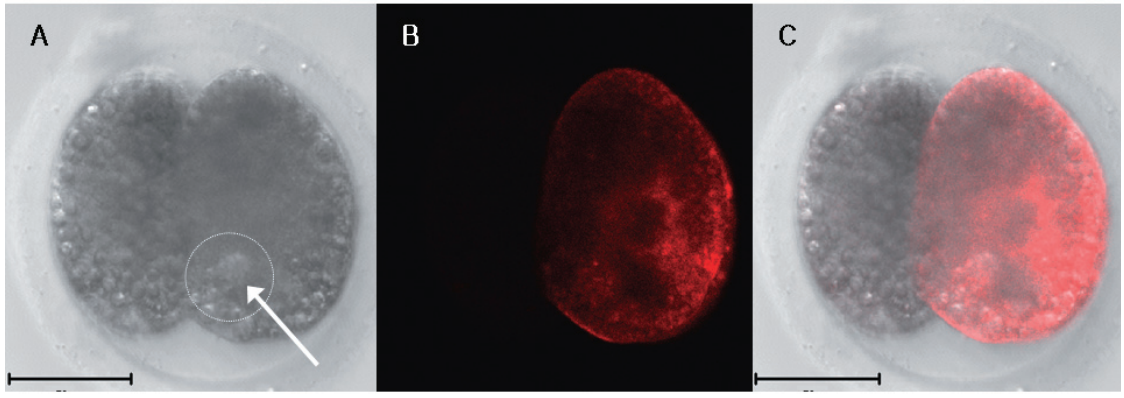

Fig. 2. Images of DiI-labeled 2-cell blastomeres. (A) Snapshot image. The arrow indicates the DiI drop. (B) Fluorescent image. Only a labeled blastomere emits red fluorescence. (C) Merged image of A and B. Bar, $40 \mu \mathrm{m}$.

A

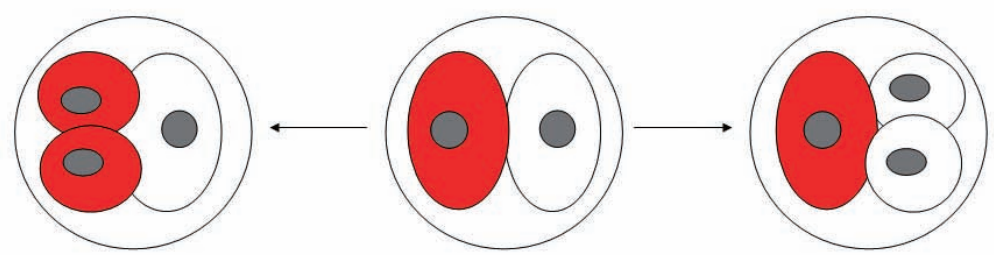

Leading

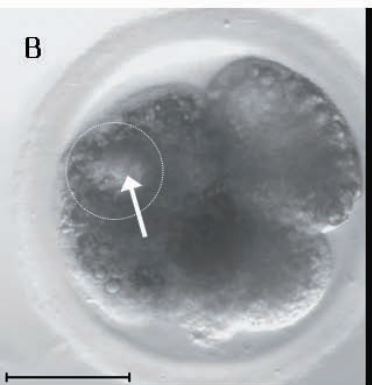

Lagging
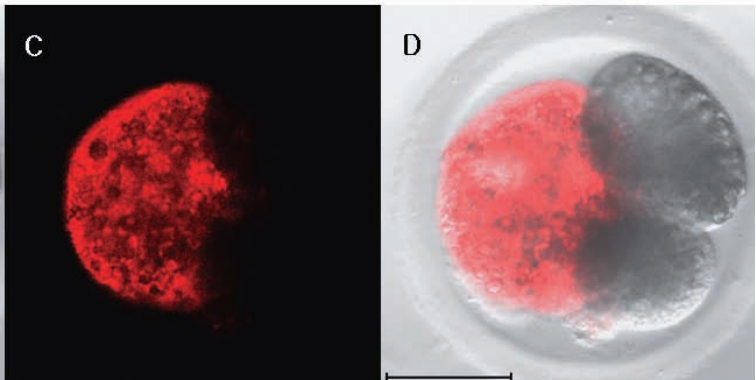

Fig. 3. (A) Illustration of the 'leading' and 'lagging' embryo groupings. If the first dividing blastomere was labeled, the embryos were included in the 'leading' group, and all other embryos were grouped as 'lagging'. (B) Snapshot image of a 'lagging' blastomere. The arrow indicates the DiI drop. (C) Fluorescent image of a 'lagging' blastomere. Only a labeled blastomere emits red fluorescence. (D) Merged image of B and C. Bar, $40 \mu \mathrm{m}$. 

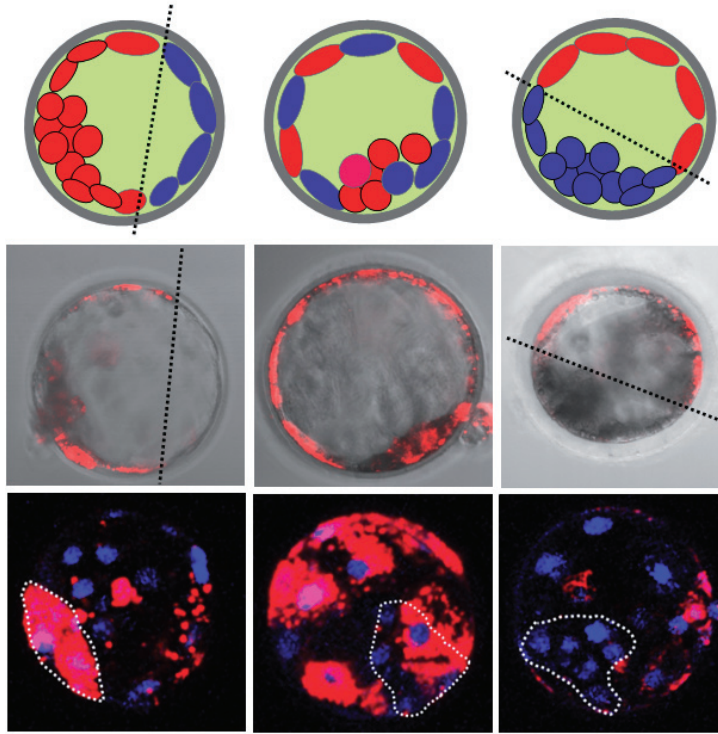

Unspecified

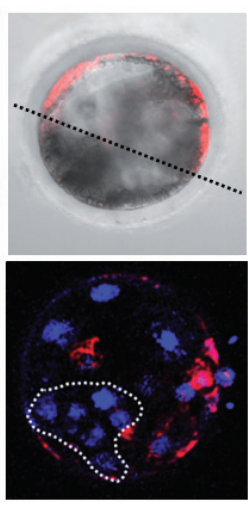

Hemi-TE

Fig. 4. Classification of the DiI-labeled parthenogenetic porcine blastocysts. Hemi-ICM: The progeny of the labeled blastomere formed the inner cell mass (ICM) and adjacent TE hemisphere in the blastocysts. Unspecified: The progeny of a labeled blastomere were distributed randomly thoughout the whole blastocyst. Hemi-TE: The progeny of a labeled blastomere formed the TE hemisphere in the blastocyst. Most cells were located in the opposite hemisphere from the ICM. To confirm the distribution of the descendants, the nuclei of the embryonic cells in the blastocysts were also stained with Hoechst\#33342 (blue); the images are presented as Z-stack pictures (bottom three). Dotted circles indicate the ICMs in the blastocysts.

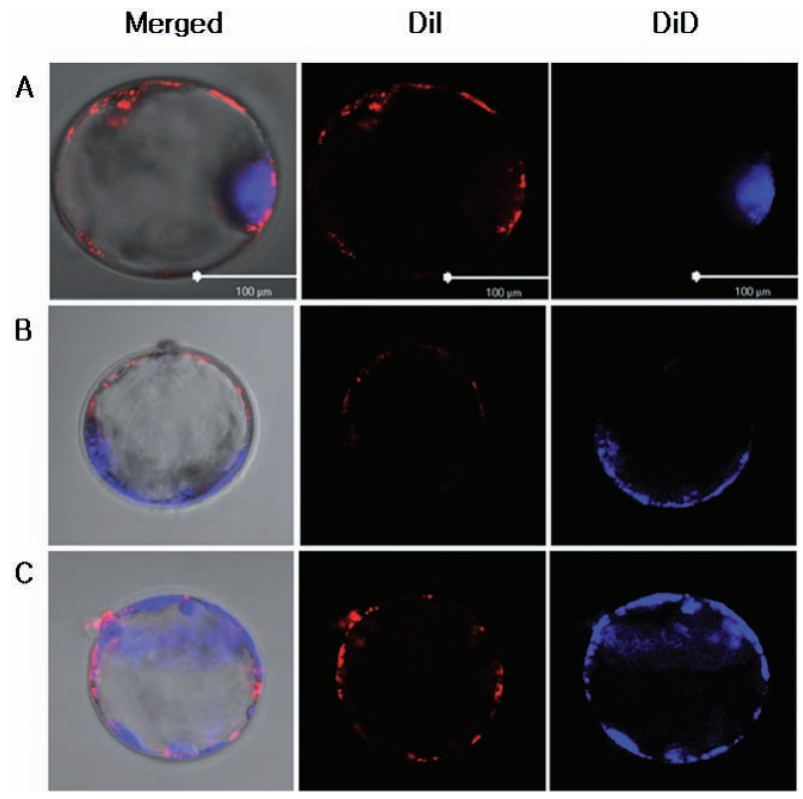

Fig. 5. Fluorescence images of blastocysts after bilateral labeling of 2-cell blastomeres with DiI (red) and DiD (blue). (A) The progeny of labeled 2-cell blastomeres emitted fluorescence in the ICM or TE exclusively. (B) The progeny of labeled 2-cell blastomeres emitted fluorescence in each hemisphere of the blastocyst. (C) The progeny of labeled blastomeres were distributed randomly throughout the whole blastocyst. Bar, $100 \mu \mathrm{m}$.
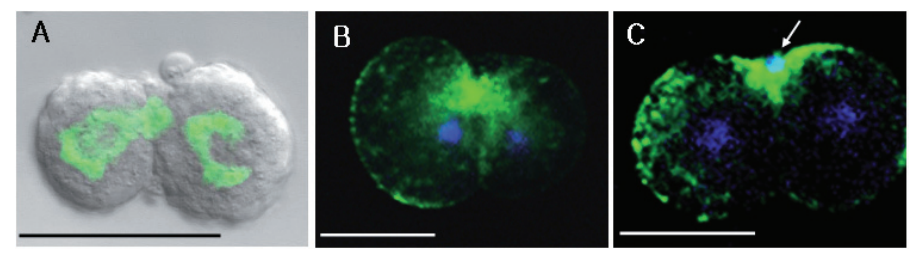

Fig. 6. Distribution of mitochondria in murine and porcine 2-cell parthenotes. Green: mitochondria. Blue: nuclei of the embryo. (A) Mitochondria were distributed around nuclei in murine embryos. (B) The mitochondria remained around the cleavage furrow in porcine embryos. (C) Biased mitochondrial distribution was found in some porcine embryos. The blue spots around the cleavage furrow are artifacts from the remnants of the zona pellucida (arrow). Bar, $50 \mu \mathrm{m}$.

and the blastocysts were divided into three groups (HemiICM, Unspecified and Hemi-TE). The blastocysts derived from the 2-cell embryos bilaterally labeled with DiI and DiD were grouped as 'ICM or TE only', 'Hemi-ICM/TE' and Unspecified. The classification details are described in Fig. 4 and Fig. 5. Labeled but dead blastomeres in the blastocysts and embryos showing unclear labeling were strictly excluded from the experiments.
The aim of the second stage of the experiment was to determine the distribution of mitochondria in 2-cell embryos. Parthenogenetic murine and porcine embryos at the 1-cell stage were pre-labeled with $500 \mathrm{nM}$ of a vital, mitochondrion-specific fluorochrome (Mitotracker Green FM, Molecular Probes, Eugene, OR, U.S.A.) for $30 \mathrm{~min}$ either at $37^{\circ} \mathrm{C}$ (murine) or $38.5^{\circ} \mathrm{C}$ (porcine) in a $5 \% \mathrm{CO}_{2}$ incubator. Porcine embryos were also stained with $10 \mu \mathrm{g} /$ 
$\mathrm{ml}$ Hoechst\#33342 to label the nuclei. The labeled embryos were cultured for $24 \mathrm{hr}$, and after zona pellucida removal and washing in PBS, 2-cell murine and porcine parthenotes were mounted onto glass slides and observed using a confocal microscope system. If the mitochondria were distributed around the nucleus, they were classified as 'Perinuclear', while mitochondria that remained around the cleavage furrow were classified as 'Cleavage furrows'. Bias of the mitochondrial distribution of each blastomere in 2-cell embryos was also assessed (Fig. 6). The data were expressed as means \pm SEM.

Animal ethics: All animal experiments were approved and performed under the guidelines of the Institutional Animal Care and Use Committee of Seoul National University.

\section{RESULTS}

Distribution of blastomere progeny in parthenogenetic porcine embryos: One hundred and ninety-eight 2-cell parthenogenetic porcine embryos were successfully stained by DiI injection, and $23.4 \%$ (67/286) of the embryos developed into blastocysts. In $60.5 \%$ (23/38) of the blastocysts in the group referred to as lagging, the progeny of labeled blastomeres formed an ICM and adjacent TE hemisphere in the blastocysts (Hemi-ICM), while 62.1\% (18/29) of the blastocysts in the group referred to as leading contained progeny of the labeled blastomeres that were distributed only to the TE (mostly in the opposite hemisphere of the ICM; HemiTE). The rest of the lagging $(36.8 \%, 24 / 38)$ and leading (34.5\%, 10/29) groups exhibited varying distributions within the blastocysts (Unspecified; Table 1). Bilateral labeling with DiI and DiD of each blastomere in 2-cell embryos reduced the rate of blastocysts $(27.3 \%, 60 / 220)$ but did not affect the distributional tendency of the progeny of 2-cell stage blastomeres. Only 33.3\% (20/60) of blastocysts exhibited an unspecified progeny distribution, while the other blastocysts showed biased distributions (66.6\%, 40/ 60; Table 2).

Distribution of mitochondria in murine and porcine 2cell parthenotes: The mitochondria of all the 2-cell murine parthenotes were distributed around the nucleus (100\%, 20/ 20 ), while the majority of mitochondria in the porcine parthenotes remained around the cleavage furrow (78.6\%, 33/ 42). Biased mitochondria distributions were found in $38.1 \%$

Table 1. Distribution of blastomere progeny in parthenogenetic porcine embryos after labeling with red fluorescent dye, DiI, of single blastomeres from 2-cell embryos ${ }^{\mathrm{a})}$

\begin{tabular}{|c|c|c|c|c|c|c|}
\hline \multirow{2}{*}{$\begin{array}{c}\text { Total } \\
\text { number of } \\
\text { 2-cell } \\
\text { embryos }\end{array}$} & \multirow{2}{*}{ Group $^{\text {b) }}$} & \multirow{2}{*}{$\begin{array}{c}\text { Number (\%) } \\
\text { embryos } \\
\text { grouped }\end{array}$} & \multirow{2}{*}{$\begin{array}{l}\text { Number (\%) } \\
\text { blastocysts }\end{array}$} & \multicolumn{3}{|c|}{$\begin{array}{l}\text { Distribution of the progeny of the } \\
\text { blastomeres }(\%)^{\mathrm{c})}\end{array}$} \\
\hline & & & & Hemi-ICM & Unspecified & Hemi-TE \\
\hline \multirow[t]{2}{*}{286} & Lagging & 171 (59.8) & 38 (22.2) & $23(60.5)$ & $14(36.8)$ & $1(2.6)$ \\
\hline & Leading & $115(40.2)$ & 29 (25.2) & $1(3.4)$ & $11(34.5)$ & $18(62.1)$ \\
\hline
\end{tabular}

a) Eight replicates.

b) Lagging: 2-cell embryos in which the blastomere from the second division was labeled; Leading: 2-cell embryos in which the blastomere from the first division was labeled.

c) A detailed classification of the blastomeric distribution is described in Fig. 4.

Table 2. Distribution of the blastomere progeny in parthenogenetic porcine embryos after bilateral labeling with the red (DiI) and blue (DiD) fluorescent dyes of each blastomere from the 2-cell embryos ${ }^{\text {a) }}$

\begin{tabular}{|c|c|c|c|c|}
\hline \multirow{2}{*}{$\begin{array}{c}\text { Number of } \\
\text { 2-cells embryos }\end{array}$} & \multirow{2}{*}{$\begin{array}{l}\text { Number (\%) } \\
\text { blastocysts }\end{array}$} & \multicolumn{3}{|c|}{ Distribution of the progeny of the blastomeres (\%) } \\
\hline & & $\begin{array}{l}\text { ICM or TE only } \\
\text { (A) }\end{array}$ & $\begin{array}{l}\text { Hemi-ICM/TE } \\
\text { (B) }\end{array}$ & $\begin{array}{l}\text { Unspecified } \\
\text { (C) }\end{array}$ \\
\hline \multirow{2}{*}{220} & \multirow{2}{*}{$60(27.3)$} & $9(15.0)$ & $31(51.7)$ & \multirow{2}{*}{$20(33.3)$} \\
\hline & & \multicolumn{2}{|c|}{$(\mathrm{A})+(\mathrm{B})=40(66.7)$} & \\
\hline
\end{tabular}

a) Five replicates.

b) A detailed classification of the blastomeric distribution is described in Fig. 5.

Table 3. Differential distribution of mitochondria in murine and porcine 2-cell parthenotes ${ }^{\text {a) }}$

\begin{tabular}{ccccc}
\hline Group & $\begin{array}{c}\text { Number } \\
\text { 2-cell } \\
\text { parthenotes }\end{array}$ & \multicolumn{2}{c}{$\begin{array}{c}\text { Distribution of mitochondria } \\
(\% \text { mean } \pm \text { SEM })^{\text {b) }}\end{array}$} & $\begin{array}{c}\text { Biased distribution of } \\
\text { mitochondria }\end{array}$ \\
\cline { 2 - 3 } Murine & 20 & $\begin{array}{c}\text { Perinuclear } \\
(\% \text { mean } \pm \text { SEM })^{\text {b) }}\end{array}$ \\
\hline Porcine & 42 & $9(21.4 \pm 2.5)$ & $33(78.6 \pm 4.6)$ & $0(0.0)$ \\
\hline
\end{tabular}

a) Five replicates.

b) A detailed classification of the mitochondrial distribution is shown in Fig. 6 . 
(16/42) of the 2-cell porcine embryos (Table 3).

\section{DISCUSSION}

In the present study, the Em-Ab axis of the porcine blastocyst was investigated. To prevent topological change of two or more apposing pronuclei in the egg center resulting from polyspermy and to avoid the influences of the fertilization cone, which indicates the sperm entry position, parthenogenetic porcine embryos were used in the present experiments. The blastomere-labeling experiment indicated that in 2-cell porcine parthenotes, the first blastomere to be cleaved, which we referred to as the leading blastomere, formed the distal TE $(=\mathrm{Ab})$ and the second blastomere to be cleaved, which we called the lagging blastomere, formed the rest of the blastocyst, including the ICM and adjacent TE (=Em). Contrary to the reports showing no tendency for the first two parthenogenetic blastomeres to follow different fates in the murine [22], this result suggests that the fate of early porcine embryos exists as an intrinsic property of the oocyte itself.

To trace the fates of each blastomere, membrane soluble fluorescent dyes DiI and DiD were used to label blastomeres at the 2-cell stage. Although some investigators have claimed that intracytoplasmic injection of lineage-tracing substances may disturb the normal pattern of development by delaying the cell division cycle of the injected blastomeres [1, 20], we injected dye directly into the cytoplasm of porcine blastomeres. It is known that porcine oocytes have greater lipid content than other domestic animals [3]. Due to the high amount of endogenous lipids, porcine oocytes and early embryos appear dark. An immature porcine oocyte typically contains $156 \mathrm{ng}$ of lipid [17], while mouse oocytes contain only $4 \mathrm{ng}$ [16]. Thus, we assumed that the lipid-rich cytoplasm of early porcine embryos would help the diffusion of lipophilic fluorescent dye. However, in the preliminary experiments of this study, we were unable to stain the cytoplasm of the blastomere by positioning the oil containing the dye as is generally done in mice. This limitation may be a characteristic of the porcine species or may be caused by the in vitro maturation procedure. Due to this technical limitation, the only way to label the blastomeres with DiI and DiD was by direct injection of an oil drop containing the dye. If intracytoplasmic dye injection did indeed delay the blastomeric cell cycle, most blastomeres labeled by injection might be scored as 'lagging'. However, $40.2 \%(115 / 286)$ of the progeny of the 2cell embryos were scored as 'leading', indicating that intracytoplasmic injection did not completely perturb the cell division cycle in pigs, although it was partly affected by the injection (see Table 1). To completely exclude the possible effect of cytoplasmic injection on cell cycle delay, we injected each blastomere of some 2-cell embryos with different colored dyes (DiI, red, and DiD, blue). The patterning of the progenies of the bilaterally-labeled 2-cell embryonic cells was similar to the patterning of those that were unilaterally labeled as shown in Fig. 5. Piotrowska and
Zernicka-Goetz (2001) claimed that the blastomere in the two-cell stage embryo that inherits the SEP divides sooner, contributes preferentially to the ICM and is not an intrinsic property of the oocyte itself as they found that there was no tendency for the first two parthenogenetic blastomeres to follow different fates [22]. However, recent time-lapse recording studies have shown that the first cleavage plane is not prepatterned [9]. In our preliminary study, we also observed unbiased contribution of the first two blastomeres (data not shown). Recently, two research groups have claimed that the blastocyst axis aligns with the shape of the zona pellucida [15, 18], whereas Gardner (2007) maintains that the axis is specified before blastulation and independently of the zona pellucida. Interestingly, our data showed that the blastomere that divided earlier tended to contribute to the trophectoderm. This finding, contrary to previous notions [21, 22], may have resulted from species differences (murine versus porcine) or from the source of the oocytes (in vivo versus in vitro).

The porcine oocyte contains 39-fold higher lipid content than that found in the mouse oocyte $[16,17]$. Early porcine embryos showing large cytoplasmic lipid droplets may have different metabolic pathways compared with their murine counterparts. These droplets are considered to play important roles in energy metabolism during oocyte maturation, fertilization and early embryonic development [11]. However, it has also been reported that endogenous triglycerides act as metabolic substrates during in vitro maturation only and not during early embryonic development in pigs [25]. The direct role of the high lipid content in early porcine embryos remains to be elucidated. In vitro maturation also changes the features of lipid droplets reflecting different energy statuses [11]. Hence, the differences in the contents or features of cytoplasmic lipids between in vivo derived murine oocytes and in vitro matured porcine oocytes might change the patterning of the Em-Ab axis. In some 2-cell embryos, one blastomere contains more lipids than the other; we attempted to analyze the relationship between the lipid content and cell division cycle in the blastomere. Unfortunately, no data with objective validity could be obtained (data not shown).

The distribution of active mitochondria may be indicative of the energy or ion requirements of many key events during oocyte maturation, fertilization and early embryonic development [26]. We hypothesized that the mitochondrial distributions of each blastomere in 2-cell parthenotes may differ. If one blastomere of a 2-cell embryo requires more energy than the other, more active mitochondria possibly exist in the fast dividing cells. Interestingly, we observed some biased distributions of mitochondria at the late 2-cell stage in porcine parthenotes. This result differs from that of a previous study using fertilized embryos [26]. According to this previous report, mitochondrial aggregation was found in the region in which the blastomeres were in contact at the early cleavage stage and the mitochondria were clustered around the blastomeric nuclei thereafter. However, we were unable to find this typical mitochondrial cluster around 
blastomeric nuclei in any 2-cell embryos from the early to late stages. This may be a defect resulting from parthenogenetic activation or failure to examine the embryos at the exact late 2-cell stage. Unlike fertilized porcine embryos, biased distribution of mitochondria in porcine 2-cell parthenotes may facilitate the following of different fates in the first two parthenogenetic blastomeres. Unfortunately, since the embryos could not be developed after fixation for visualization, we were unable to examine whether mitochondria-rich embryos developed more quickly.

Taken together, our findings indicated that the porcine 2cell parthenote is likely to show Em-Ab axis formation from its preimplantational development. However, the patterning we saw was completely different than those observed in previous murine embryo studies [21, 22]. The first two blastomeres of the mammalian embryo are totipotent, meaning that they contain the complete set of information needed for normal body and placental formation [2]. Hence, if prepatterning does exist, the axis of polarity of the murine embryo at the preimplantation stage may not occur at the molecular level. Instead, prepatterning may be related to the differing energy metabolisms of each blastomere in the 2-cell embryo. Biased lipid and mitochondrial distributions may be candidate cause of this event. The uneven distribution of lipids and mitochondria may have originated from parthenogenetic activation and/or in vitro maturation as well as the species specificity in the present study.

In conclusion, this study suggests that the fate of an individual blastomere of the porcine parthenogenetic embryo at the 2-cell stage may be an intrinsic property of the oocyte itself. The 'leading' blastomere of the 2-cell porcine parthenote formed the distal TE (Ab part), while the 'lagging' blastomere formed the rest of the blastocyst, including the ICM and surrounding TE (Em part). A biased mitochondria distribution in each blastomere of the 2-cell parthenogenetic porcine embryo may contribute partly to this event. However, further studies are required to determine whether this event is related to embryonic energy metabolism or molecular mechanisms. The Em-Ab polarity of in vivo derived porcine embryos remains to be elucidated.

ACKNOWLEDGMENTS. This study was supported by Korea Science and Engineering Foundation (KOSEF) grants funded by the Government of the Republic of Korea (MOST; M10641000001-06N4100-00110 and R01-2007000-10316-0).

\section{REFERENCES}

1. Alarcon, V. B. and Marikawa, Y. 2003. Deviation of the blastocyst axis from the first cleavage plane does not affect the quality of mouse postimplantation development. Biol. Reprod. 69: $1208-1212$.

2. Alarcon, V. B. and Marikawa, Y. 2005. Unbiased contribution of the first two blastomeres to mouse blastocyst development. Mol. Reprod. Dev. 72: 354-361.

3. Cran, D. G. 1987. The distribution of organelles in mammalian oocytes following centrifugation prior to injection of foreign
DNA. Gamete Res. 18: 67-76.

4. Davies, T. J. and Gardner, R. L. 2002. The plane of first cleavage is not related to the distribution of sperm components in the mouse. Hum. Reprod. 17: 2368-2379.

5. Fujimori, T., Kurotaki, Y., Miyazaki, J. and Nabeshima, Y. 2003. Analysis of cell lineage in two- and four-cell mouse embryos. Development 130: 5113-5122.

6. Gardner, R. L. 1997. The early blastocyst is bilaterally symmetrical and its axis of symmetry is aligned with the animalvegetal axis of the zygote in the mouse. Development 124: 289-301.

7. Gardner, R. L. 2001. Specification of embryonic axes begins before cleavage in normal mouse development. Development 128: 839-847.

8. Gore, A. V., Maegawa, S., Cheong, A., Gilligan, P. C., Weinberg, E. S. and Sampath, K. 2005. The zebrafish dorsal axis is apparent at the four-cell stage. Nature 438: 1030-1035.

9. Hiiragi, T. and Solter, D. 2004. First cleavage plane of the mouse egg is not predetermined but defined by the topology of the two apposing pronuclei. Nature 430: 360-364.

10. Kessler, D. S. and Melton, D. A. 1995. Induction of dorsal mesoderm by soluble, mature Vg1 protein. Development 121: 2155-2164.

11. Kikuchi, K., Ekwall, H., Tienthai, P., Kawai, Y., Noguchi, J., Kaneko, H. and Rodriguez-Martinez, H. 2002. Morphological features of lipid droplet transition during porcine oocyte fertilisation and early embryonic development to blastocyst in vivo and in vitro. Zygote 10: 355-366.

12. Kimelman, D. 2006. Squinting at the zebrafish axis. Dev. Cell 10: 6-7.

13. Koo, J.-M., Won, C., Min, B.-M. and Roh, S. 2005. Development of a chemically defined in vitro maturation system for porcine oocytes: application for somatic cell nuclear transfer. Int. J. Oral. Biol. 30: 131-134.

14. Kouba, A. J., Abeydeera, L. R., Alvarez, I. M., Day, B. N. and Buhi, W. C. 2000. Effects of the porcine oviduct-specific glycoprotein on fertilization, polyspermy, and embryonic development in vitro. Biol. Reprod. 63: 242-250.

15. Kurotaki, Y., Hatta, K., Nakao, K., Nabeshima, Y. and Fujimori, T. 2007. Blastocyst axis is specified independently of early cell lineage but aligns with the ZP shape. Science 316: 719-723.

16. Loewenstein, J. E. and Cohen, A. I. 1964. Dry mass, lipid content and protein content of the intact and zona-free mouse ovum. J. Embryol. Exp. Morphol. 12: 113-121.

17. McEvoy, T. G., Coull, G. D., Broadbent, P. J., Hutchinson, J. S. and Speake, B. K. 2000. Fatty acid composition of lipids in immature cattle, pig and sheep oocytes with intact zona pellucida. J. Reprod. Fertil. 118: 163-170.

18. Motosugi, N., Bauer, T., Polanski, Z., Solter, D. and Hiiragi, T. 2005. Polarity of the mouse embryo is established at blastocyst and is not prepatterned. Genes Dev. 19: 1081-1092.

19. Motosugi, N., Dietrich, J., Polanski, Z., Solter, D. and Hiiragi, T. 2006. Space asymmetry directs preferential sperm entry in the absence of polarity in the mouse oocyte. PLOS Biol. 4: 1-6.

20. Piotrowska, K., Wianny, F., Pedersen, R. A. and ZernickaGoetz, M. 2001. Blastomeres arising from the first cleavage division have distinguishable fates in normal mouse development. Development 128: 3739-3748.

21. Piotrowska, K. and Zernicka-Goetz, M. 2001. Role for sperm in spatial patterning of the early mouse embryo. Nature 409: 517-521.

22. Piotrowska, K. and Zernicka-Goetz, M. 2002. Early patterning 
of the mouse embryo--contributions of sperm and egg. Development 129: 5803-5813.

23. Plusa, B., Hadjantonakis, A. K., Gray, D., Piotrowska-Nitsche, K., Jedrusik, A., Papaioannou, V. E., Glover, D. M. and Zernicka-Goetz, M. 2005. The first cleavage of the mouse zygote predicts the blastocyst axis. Nature 434: 391-395.

24. Rossant, J. 1976. Postimplantation development of blastomeres isolated from 4- and 8-cell mouse eggs. J. Embryol. Exp. Morphol. 36: 283-290.

25. Sturmey, R. G. and Leese, H. J. 2003. Energy metabolism in pig oocytes and early embryos. Reproduction 126: 197-204.

26. Sun, Q. Y., Wu, G. M., Lai, L., Park, K. W., Cabot, R., Cheong, H. T., Day, B. N., Prather, R. S. and Schatten, H. 2001. Translocation of active mitochondria during pig oocyte maturation, fertilization and early embryo development in vitro. Reproduction 122: 155-163.

27. Tarkowski, A. K. 1959. Experiments on the development of isolated blastomers of mouse eggs. Nature 184: 1286-1287.

28. Tarkowski, A. K. and Wroblewska, J. 1967. Development of blastomeres of mouse eggs isolated at the 4- and 8-cell stage. $J$. Embryol Exp. Morphol. 18: 155-180.

29. Waksmundzka, M., Wisniewska, A. and Maleszewski, M. 2006. Allocation of cells in mouse blastocyst is not determined by the order of cleavage of the first two blastomeres. Biol. Reprod. 75: 582-587.

30. Willadsen, S. M. 1981. The development capacity of blastomeres from 4- and 8-cell sheep embryos. J. Embryol Exp. Morphol. 65: 165-172. 